\title{
Treatment of stage I non-small cell lung cancer with CyberKnife, image-guided robotic stereotactic radiosurgery
}

\author{
SE HAN AHN ${ }^{1}$, MIN SUNG HAN ${ }^{1}$, JUNG HO YOON ${ }^{1}$, SE YONG JEON ${ }^{1}$, \\ CHEOL HYEON KIM ${ }^{1}$, HYUNG JUN YOO ${ }^{2}$ and JAE CHEOL LEE ${ }^{1}$ \\ Departments of ${ }^{1}$ Internal Medicine and ${ }^{2}$ Radiation Oncology, Korea Cancer Center Hospital, Seoul, Korea
}

Received September 3, 2008; Accepted December 29, 2008

DOI: $10.3892 /$ or_00000273

\begin{abstract}
The object of our study was to evaluate the clinical characteristics and outcomes of patients with stage I nonsmall cell lung cancer (NSCLC) who underwent radiosurgery using the CyberKnife, a newly developed technology to deliver radiation from multiple angles with a real-time target tracking system. A retrospective analysis of eight patients with stage I NSCLC who were treated with curative intent using the CyberKnife between 2002 and 2007 at a cancer center in Korea was performed. Among eight patients (seven men and one woman), three patients were ineligible for surgery due to poor lung function while four patients refused surgical treatment. Tumor size ranged from 19 to $50 \mathrm{~mm}$ in the maximal diameter (12 to $113 \mathrm{ml}$ in volume). The administered radiation dose varied from 36 to 54 Gy in three fractions. All of the patients tolerated the treatment very well without any significant side effects. Complete response was achieved and was sustained for almost two years in one male patient until the patient died from a cerebrovascular accident. Seven patients showed radiographic partial response at 1-3 months. Re-growth of tumor at the treated site was observed in only one patient demonstrating an excellent local control rate, although systemic spread or regional lymph node metastasis of disease occurred in six patients during followup. CyberKnife treatment is very safe and is able to achieve a high local control rate, suggesting its role as a reasonable alternative therapeutic modality in early lung cancer.
\end{abstract}

\section{Introduction}

Surgery is the treatment of choice for early stage non-small cell lung cancer (NSCLC). Surgical resection of stage IA and

Correspondence to: Dr Jae Cheol Lee, Department of Internal Medicine, Korea Cancer Center Hospital, 215-4, Gongneung-dong, Nowon-gu, Seoul 139-706, Korea

E-mail: jclee@kcch.re.kr

Key words: CyberKnife, early lung cancer, non-small cell lung cancer, radiotherapy, robotic radiosurgery
IB NSCLC results in a 5-year survival rate of $\sim 60-70 \%$ (1). However, it is often difficult to recommend surgery to some patients because of accompanying medical illness such as severe chronic obstructive pulmonary disease with very limited lung reserve. Actually, the number of lung cancer patients with comorbid conditions appears to be on the increase due to an increasing number of elderly patients at the time of diagnosis. Moreover, there are patients who refuse to undergo surgery due to emotional or religious reasons. For such patients, conventional radiotherapy with a dose of 50-80 Gy has been used with 2- and 5-year survival rates of 39 and $13 \%$, respectively (2). Recent studies have suggested that precisely targeted radiosurgery with a higher dose may be a reasonable alternative to overcome limitations of conventional radiotherapy such as damage to normal tissues or high local failure (2-7). The CyberKnife (image-guided robotic stereotactic radiosurgery, IGR-SRS) system is a newly developed technology to deliver radiation from multiple angles with a real-time target tracking system. The accuracy of target localization and dose delivery can be achieved through combining real-time, periodic X-ray imaging of implanted metallic markers (called fiducials) within the tumor. The accuracy of the tracking system is within $1.5 \mathrm{~mm}$ of the planning treatment volume (PTV) margins and allows the tumor to be destroyed precisely $(8,9)$. Although this system is increasingly being used, there have been a few reports from a very limited number of centers about the results of CyberKnife treatment for early lung cancer (8-10). We evaluated the clinical characteristics and outcomes of patients with stage I NSCLC who underwent radiosurgery using the CyberKnife at a cancer center in Korea.

\section{Materials and methods}

A retrospective analysis of eight patients with stage I NSCLC who were treated with curative intent with the CyberKnife ${ }^{\circledR}$ system between 2002 and 2007 at a tertiary referral center for cancer in Korea was performed. An oncologist, radiologist, pulmonologist and a thoracic surgeon evaluated each patient. Primary pulmonary tumors were irradiated with total doses of 36-54 Gy, delivered in three fractions for three days. The prescribing PTV was intended to enclose $100 \%$ of the gross tumor volume (GTV). The PTV was outlined with 6-8 $\mathrm{mm}$ beyond the GTV to cover microscopic infiltration and uncertainties caused by respiratory motion. The 

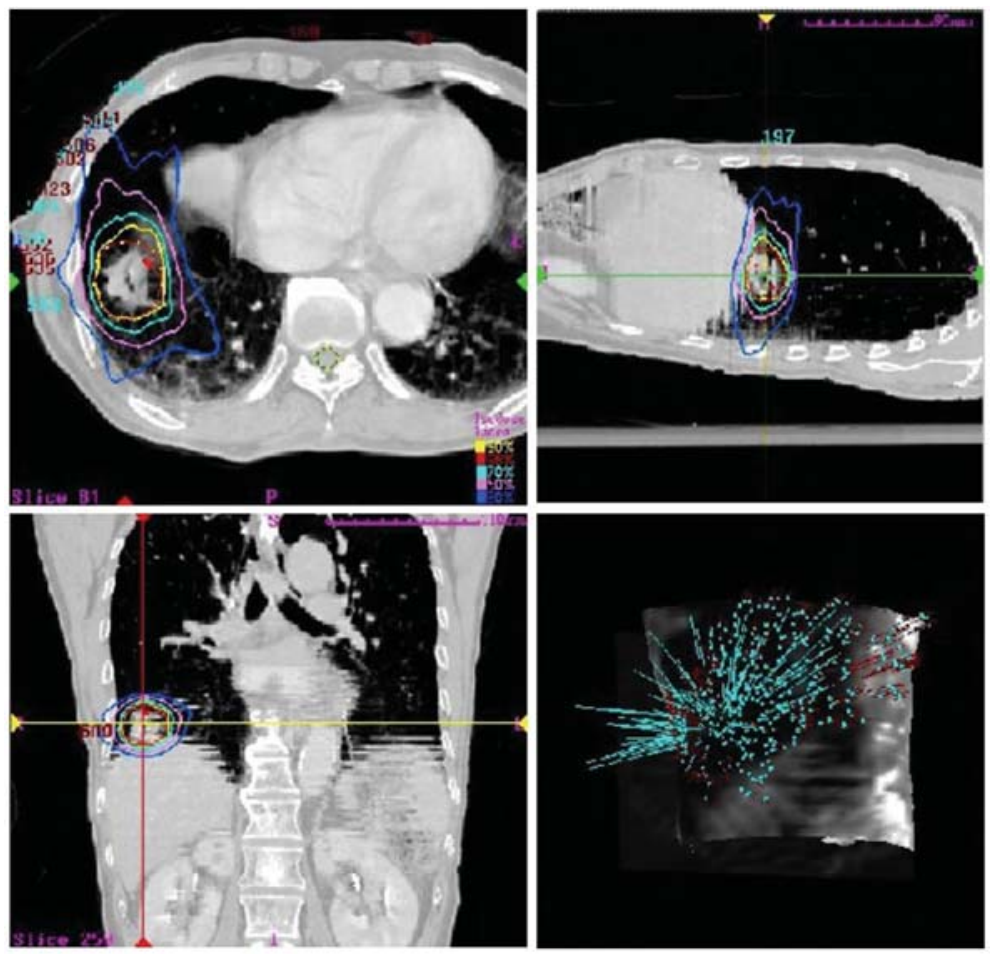

Figure 1. Treatment plan for the CyberKnife with a total dose of $45 \mathrm{~Gy} /$ three fractions to $20 \mathrm{~cm}^{3}$ of a non-small cell lung cancer (patient no. 3 ).

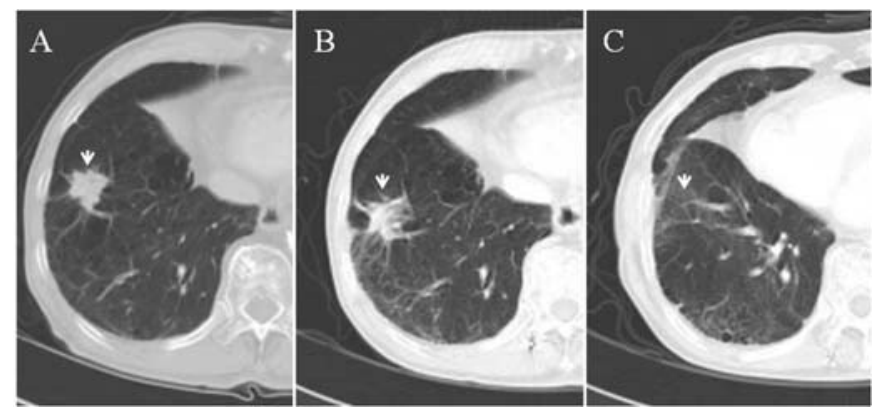

Figure 2. (A) A chest CT scan before treatment shows a $2.5 \mathrm{~cm}$-sized, lobulated nodule in the right upper lobe. (B) The size of the lung nodule decreased at 1 month after radiosurgery with the CyberKnife. (C) The lung cancer on the right lower lobe has nearly completely resolved at 10 months after CyberKnife treatment.

prescribed dose was typically up to the 78 to $83 \%$ isodose line and the heterogeneous higher dose was delivered to the center of the tumor (Fig. 1). However, the total dose was determined by consideration of the tumor size, location, and proximity to vital structures such as the heart, great vessels, major bronchi, spinal cord, esophagus, brachial plexus and diaphragm. The presence of comorbid disease and any recommendations of the radiation oncologist were considered of importance. The institutional review board of our hospital reviewed and approved the current study. The signed informed consents were obtained from patients on follow-up.

\section{Results}

All patients (seven men and one woman), with ages ranging from 68 to 80 years, were diagnosed with stage I NSCLC.
Tumor size ranged from 19 to $50 \mathrm{~mm}$ in the maximal diameter (12 to $113 \mathrm{ml}$ in volume). Since the treatment has evolved over several years, the administered dose varied from 36 to 54 Gy, with a tendency to increase with the passage of time. Three patients were ineligible for surgery due to poor lung function (forced expiratory volume in one second, FEV1, was 1.11, 1.37 and $0.7 \mathrm{~L}$ ) while four patients refused surgical treatment.

All of the patients tolerated the treatment very well without any significant side effects. Some patients just felt mild malaise. Mild, asymptomatic radiation pneumonitis developed in one patient; however, it resolved without any specific treatment. There were no complaints of worsening dyspnea after CyberKnife treatment. No pneumothorax occurred after fiducial placement.

Complete response was achieved and was sustained for almost two years in one patient (Fig. 2). Seven patients showed radiographic partial response at 1-3 months. Followup ranged from 5 to 49 months. Re-growth of tumor at the treated site was observed in only one patient that demonstrated excellent local control rate although systemic spread or a regional lymph node metastasis occurred in five patients during follow-up. Two patients died of a cerebrovascular accident without any relation to the malignancy or treatment. Sepsis developed during chemotherapy, leading to death in one patient with a recurred tumor in the bone. One patient succumbed to cancer progression. Data summarizing the demographic characteristics, clinical manifestations, treatment and follow-up information are listed in Table I.

\section{Discussion}

To avoid significant morbidity and mortality in patients with early lung cancer with serious other medical problems, 
ONCOLOGY REPORTS 21: 693-696, 2009

695

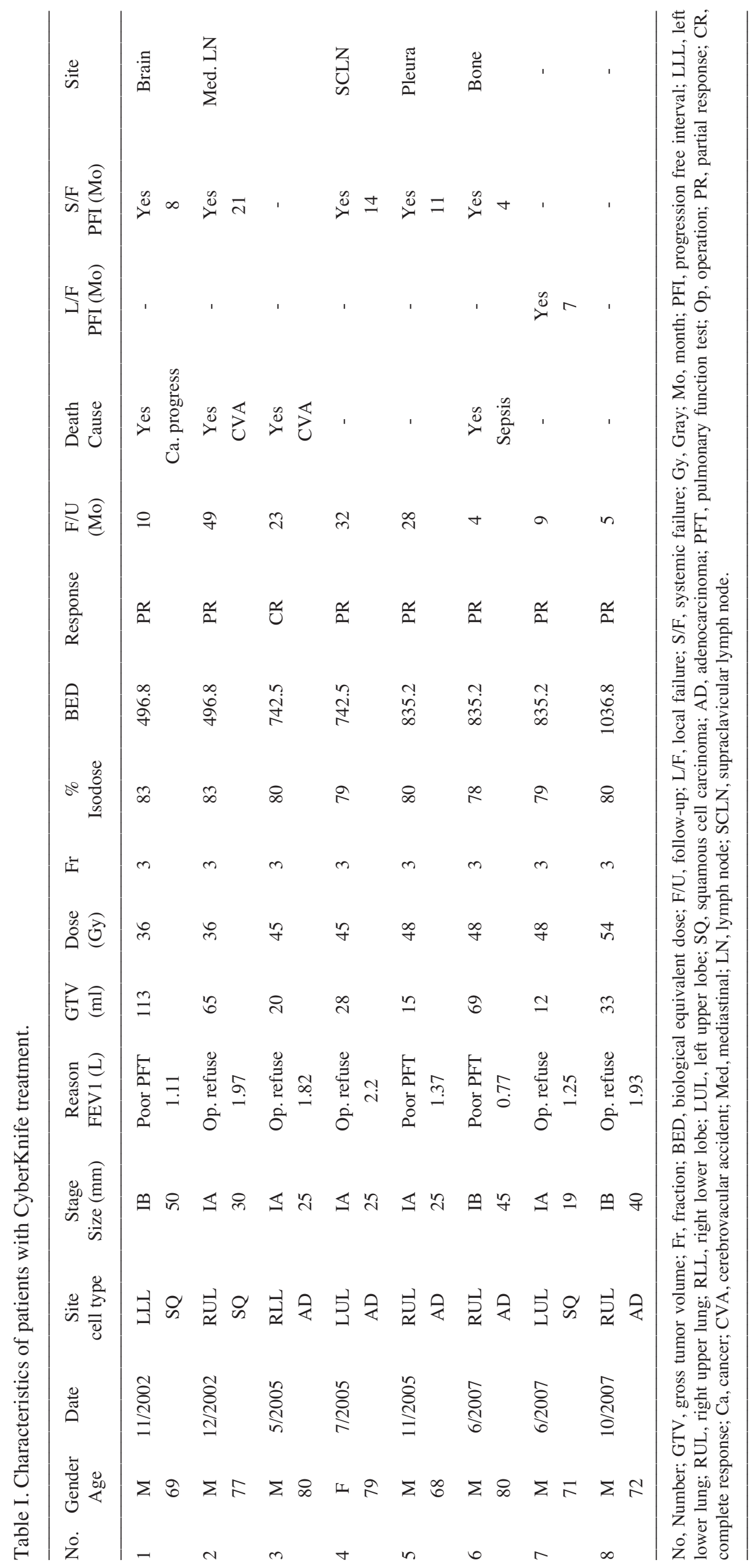


radiotherapy is considered the modality of choice (11-13). However, conventional radiotherapy can cause damage to the lung and adjacent structures producing unwanted adverse effects such as radiation pneumonitis or esophagitis that sometimes lead to a worse performance of patients (2-7). In addition, the local failure rate is higher than that of surgical resection, in part because of limited doses and fractionation of the administered radiation to circumvent side effects. Fractionation might allow repopulation of tumor cells during rest time limiting the efficacy of radiotherapy (14). Stereotactic radiotherapy, which was designed to deliver a higher dose to the tumor mass in small fractions without collateral injury, has been an attractive alternative modality to address these issues. However, a limitation still exists due to difficulties for targeting tumors that move with respiration (2-7). The CyberKnife was developed to target the moving tumor with respiration more precisely with the use of a real-time target tracking system.

Whyte et al first reported the results of CyberKnife treatment for lung cancer (15). However, the local control rate was not good, possibly because the investigators used a lower dose of <30-34 Gy in a single fraction due to little experience with the use of CyberKnife treatment of lung cancer at an early stage. Similarly, we started with total dose of 36 Gy at our CyberKnife center and found we could increase the treatment dose to $54 \mathrm{~Gy}$ in 2007 after confirming the safety of the method. We are now planning further dose escalation. Although the appropriate dose for the CyberKnife treatment for early stage NSCLC does not seem to be established as yet, Brown et al showed that a total dose up to $60 \mathrm{~Gy}$ was safe (10). Until now, there has been no report describing serious side effects after CyberKnife treatment of the lung.

Brown et al demonstrated the excellent efficacy of CyberKnife treatment with a complete response in nine of 19 patients (10). We also demonstrated a good local control rate as there was only one progression at the treatment site, even though a majority of responses was partial, which might be caused by the lower dose we utilized. Relapse outside the radiation site including the regional lymph nodes was higher than expected in our study, which we were unable to explain. A definite conclusion cannot be determined at this time due to the small number of enrolled patients and the short duration of follow-up.

The recurrence or persistence of disease may be related to microinvasion beyond the PTV or inappropriate PTV definition. Although it was difficult to identify a proper study defining the PTV margin set up beyond the GTV, Giraud et al reported that malignant cells were found at $6-8 \mathrm{~mm}$ beyond the GTV in $~ 95 \%$ of cases (16). Brown et al used a margin of $8-10 \mathrm{~mm}$ beyond the GTV for early stage NSCLC and 3-5 $\mathrm{mm}$ beyond the GTV for metastatic lesions (9). In the present study, a margin of 6-8 $\mathrm{mm}$ outside the GTV with CyberKnife treatment for lung cancer was used.

Our results showed that CyberKnife treatment is very safe and is feasible to achieve a high local control rate, suggesting its role as a reasonable alternative therapeutic modality in early lung cancer.

\section{References}

1. Mountain CF: A new international staging system for lung cancer. Chest 89: S225-S233, 1986.

2. Sibley GS, Jamieson TA, Marks LB, Anscher MS and Prosnitz LR: Radiotherapy alone for medically inoperable stage I non-smallcell lung cancer: the Duke experience. Int J Radiat Oncol Biol Phys 40: 149-154, 1998

3. McGarry RC, Papiez L, Williams M, Whitford T and Timmerman RD: Stereotactic body radiation therapy of earlystage non-small-cell lung carcinoma: phase I study. Int J Radiat Oncol Biol Phys 63: 1010-1015, 2005.

4. Onishi H, Araki T, Shirato H, et al: Stereotactic hypofractionated high-dose irradiation for stage I nonsmall cell lung carcinoma: clinical outcomes in 245 subjects in a Japanese multiinstitutional study. Cancer 101: 1623-1631, 2004.

5. Qiao X, Tullgren O, Lax I, Sirzen F and Lewensohn R: The role of radiotherapy in treatment of stage I non-small cell lung cancer. Lung Cancer 41: 1-11, 2003.

6. Timmerman R, Papiez L, McGarry R, Likes L, DesRosiers C, Frost $\mathrm{S}$ and Williams M: Extracranial stereotactic radioablation: results of a phase I study in medically inoperable stage I nonsmall cell lung cancer. Chest 124: 1946-1955, 2003.

7. Wulf J, Haedinger U, Oppitz U, Thiele W, Mueller G and Flentje M: Stereotactic radiotherapy for primary lung cancer and pulmonary metastases: a noninvasive treatment approach in medically inoperable patients. Int J Radiat Oncol Biol Phys 60: 186-196, 2004.

8. Brown WT, Wu X, Fayad F, et al: CyberKnife radiosurgery for stage I lung cancer: results at 36 months. Clin Lung Cancer 8: 488-492, 2007.

9. Brown WT, Wu X, Wen BC, et al: Early results of CyberKnife image-guided robotic stereotactic radiosurgery for treatment of lung tumors. Comput Aided Surg 12: 253-261, 2007.

10. Brown WT, Wu X, Amendola B, et al: Treatment of early nonsmall cell lung cancer, stage IA, by image-guided robotic stereotactic radioablation-CyberKnife. Cancer J 13: 87-94, 2007.

11. Hilton G: The present position relating to cancer of the lung. Results with radiotherapy alone. Thorax 15: 17-18, 1960.

12. Haffty BG, Goldberg NB, Gerstley J, Fischer DB and Peschel RE: Results of radical radiation therapy in clinical stage I, technically operable non-small cell lung cancer. Int J Radiat Oncol Biol Phys 15: 69-73, 1988.

13. Zhang HX, Yin WB, Zhang LJ, et al: Curative radiotherapy of early operable non-small cell lung cancer. Radiother Oncol 14: 89-94, 1989

14. Papiez L and Timmerman R: Hypofractionation in radiation therapy and its impact. Med Phys 35: 112-118, 2008.

15. Whyte RI, Crownover R, Murphy MJ, et al: Stereotactic radiosurgery for lung tumors: preliminary report of a phase I trial. Ann Thorac Surg 75: 1097-1101, 2003.

16. Giraud P, Antoine M, Larrouy A, et al:Evaluation of microscopic tumor extension in non-small-cell lung cancer for threedimensional conformal radiotherapy planning. Int J Radiat Oncol Biol Phys 48: 1015-1024, 2000. 